\title{
Effect of Pipeline-Soil Interaction on Subsea Pipeline Lateral Buckling Analysis
}

\author{
Yuxiao Liu ${ }^{1, a}$,* \\ ${ }^{1}$ Shandong Technology and Business University, Yantai, China \\ a63563298@qq.com
}

Keywords: submarine pipeline, lateral buckling,lateral frictional coefficient,axial frictional coefficient,yielding displacement

\begin{abstract}
Finite element method is used for post-buckling analysis of pipeline usually. In order to study effect of interaction between pipeline and seabed, FEM of pipeline exposed on seabed is built based on ANSYS, interaction between pipeline and seabed is studied, as results show that critical buckling force is increased with lateral frictional coefficient increasing, axial frictional coefficient has little effect on critical buckling force; critical buckling force is reduced with yielding displacement increasing, which can be used for engineering design.
\end{abstract}

\section{Introduction}

In recent years, there has been a rapid increase in the number of subsea pipelines transporting high pressure and high temperature hydrocarbons. Pipelines operating at high temperatures and pressures above ambient will tend to expand, due to thermal and pressure loading ${ }^{[1]}$. When the axial compressive force is large enough that the pipeline will buckle globally. For the buried pipeline, upheaval buckling will be triggered; and for the pipeline exposed on even seabed, lateral buckling will be observed ${ }^{[2]}$.

Hobbs studied the thermal buckling of pipeline induced by the transportation of high temperature fluid $^{[3-4]}$. He used perfect pipe and the model of small deflection beam-column on rigid foundation to analyze the thermal buckling behaviors of beam vertical mode and beam lateral mode. Taylor and Tran ${ }^{[5]}$ studied upheaval buckling of subsea pipeline experimentally and theoretically. Several researchers have investigated the effects of initial imperfections, but the curved pipe was assumed to be stress-free when initially deformed. Croll ${ }^{[6]}$ studied upheaval thermal buckling of subsea pipeline based on a simplified model, critical buckling force was derived, but, the pre-buckling force was not given. Yuxiao $\mathrm{Liu}^{[7]}$ studied lateral buckling of imperfect pipeline, and critical axial forces in buckling segment and away from buckling segment as well as critical temperature are deduced.

However, the global buckling formula can only be used to calculate the pipeline critical buckling load, which can not be used for post-buckling analysis of pipeline deformation, strain and moment. In order to study effect of interaction between pipeline and seabed, FEM of pipeline exposed on seabed is built based on ANSYS, interaction between pipeline and seabed is studied, as results show that critical buckling force is increased with lateral frictional coefficient increasing, axial frictional coefficient has little effect on critical buckling force; critical buckling force is reduced with yielding displacement increasing.

\section{Finite Element Model}

\subsection{Pipeline Model}

ANSYS is used to study the lateral buckling of snaked-lay pipeline. The pipeline is modeled as PIPE20 elements with plastic capability. Which stress-strain constitutional relationship is shown in Fig.1. The expression of the Ramberg-Osgood model is: 


$$
\varepsilon_{x}=\frac{\sigma_{x}}{E_{0}}\left[1+\frac{n}{1+r}\left(\frac{\sigma_{x}}{\sigma_{y}}\right)^{r}\right]
$$

Where: $\varepsilon_{x}$ and $\sigma_{x}$ are the engineering strain and stress; $E_{0}$ is the initial Young's modulus; $\sigma_{y}$ the yield stress of pipe material; $n$ and $r$ are the Ramberg-Osgood parameters.

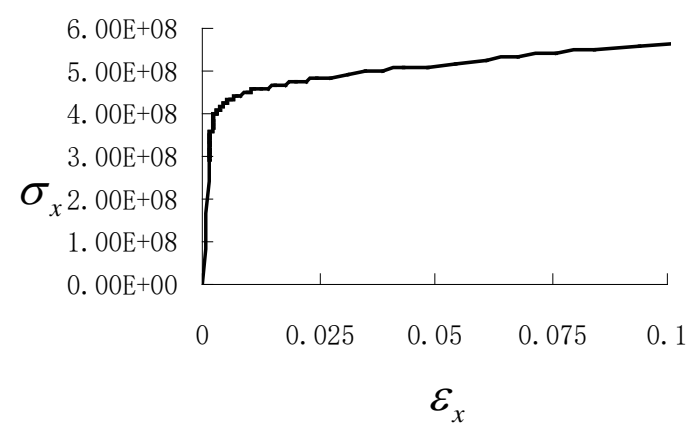

Fig.1 Stress-Strain Relationship For X65 Pipeline Steel.

\subsection{Soil Model}

The pipe-soil nonlinear interactions in the axial and lateral directions are simulated as the elastic perfectly-plastic soil springs. COMBIN39 spring element is selected. An axial spring and a lateral spring are connected to each pipe node. The seabed is simplified as flat one and spanning part of the pipeline is ignored. The pipeline is pinned in the vertical direction at each pipe node. The soil model is shown in Fig. 2, where is the maximum axial and lateral soil forces per unit length of pipe, is the yield displacements of soil springs in both directions. The relationship between yield force, friction coefficient and submerged weight along pipeline per unit can be expressed as:

$$
F=\mu W
$$

Where, $F$ is yield force; $\mu$ is friction coefficient between seabed and pipeline; $w$ is submerged weight along pipeline per unit.

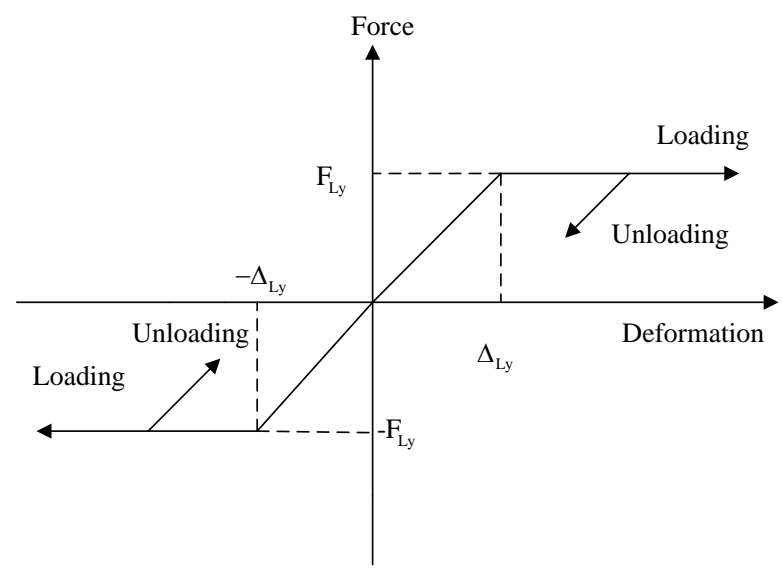

Fig.2 Model for Soil.

\subsection{Pipeline-soil interaction model}

Non-linear ideal elasto-plastic soil axial and lateral friction behavior was modeled using the COMBIN39 spring element [8-10], as Fig.3 shows. As only lateral buckling is of interest in this paper, the seabed was modeled as a flat seabed ignoring spanning parts of the pipeline. The pipeline was pinned in the vertical direction at each pipe node. FEM of pipeline is shown in Fig.4 


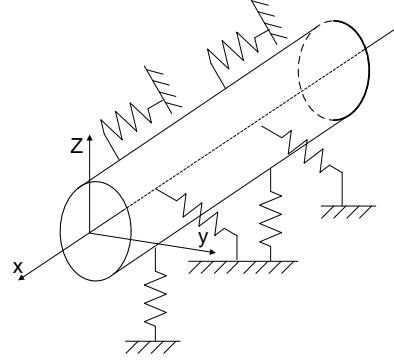

Fig.3.Pipeline-soil interaction model.

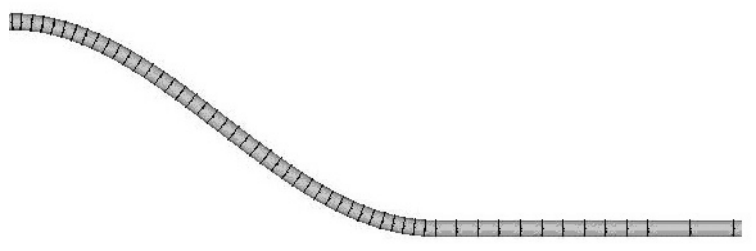

Fig. 4 Pipeline finite element model.

\section{Pipeline-Soil Interaction}

\section{1. lateral frictional coefficient}

Constant both pipeline and soil properties were used in the analyses. These properties are given in Table 1 together with the operating parameters.

Table 1 Parameters of pipeline.

\begin{tabular}{|c|c|c|}
\hline parameters & unit & value \\
\hline Pipe diameter & $\mathrm{mm}$ & 300 \\
\hline Pipe thickness & $\mathrm{mm}$ & 14 \\
\hline Thermal expansion coefficient & - & $11.7 \times 10-6$ \\
\hline Pipeline submerged weight & $\mathrm{N} / \mathrm{m}$ & 900 \\
\hline SMYS/ MPa & $\mathrm{MPa}$ & 448 \\
\hline SMTS/ MPa & $\mathrm{MPa}$ & 550 \\
\hline Derating stress at $100{ }^{\circ} \mathrm{C}$ & $\mathrm{MPa}$ & 25 \\
\hline Lateral friction coefficient & - & 0.75 \\
\hline Axial friction coefficient & - & 0.5 \\
\hline Pipeline length & $\mathrm{m}$ & 2800 \\
\hline Operating press & $\mathrm{MPa}$ & 20 \\
\hline Operating temperature & ${ }^{\circ} \mathrm{C}$ & 95 \\
\hline Ambient temperature & ${ }^{\circ} \mathrm{C}$ & 0 \\
\hline
\end{tabular}

Fig. 5 is variation of maximum lateral displacement with temperature under different axial frictional coefficient, as figure shows, critical temperature is increased with lateral coefficient increasing.

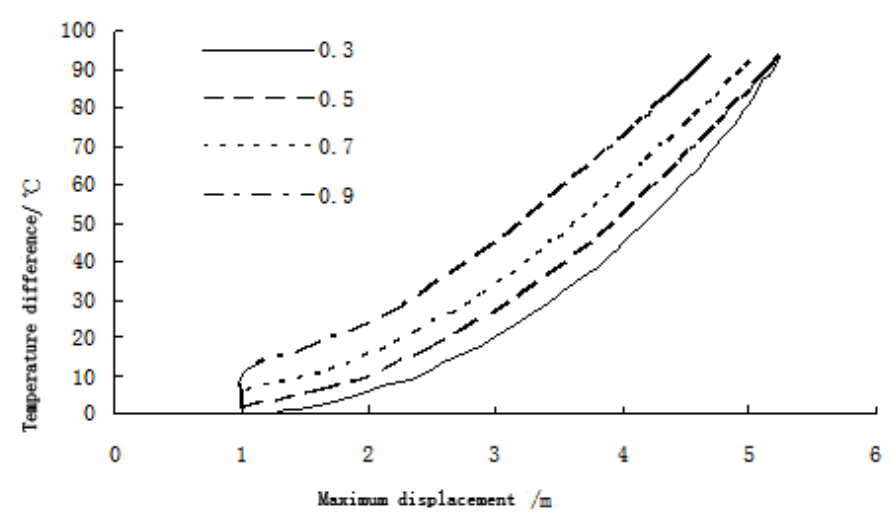

Fig. 5 Temperature vs. maximum lateral displacement for different lateral frictional coefficients. 


\subsection{Axial frictional coefficient}

Fig.6 is variation of maximum lateral displacement with temperature under different axial frictional coefficient, as figure shows, critical temperature changed little as axial frictional coefficient changed.

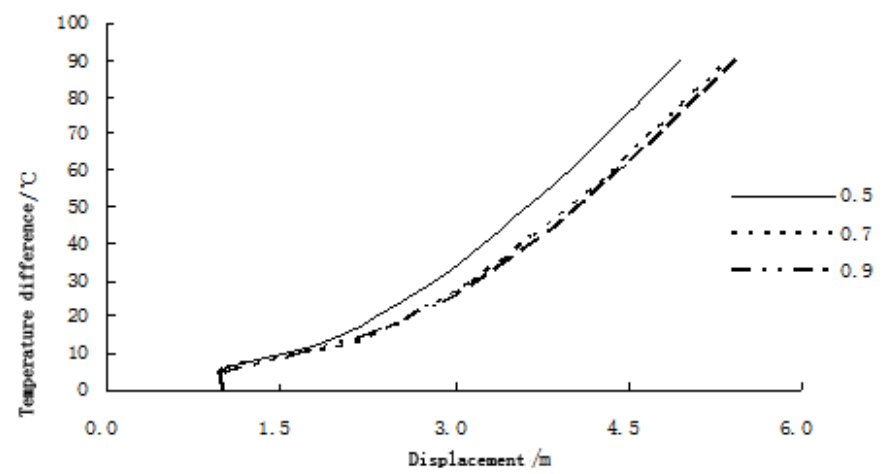

Fig. 6 Temperature vs. maximum lateral displacement for different axial frictional coefficients.

\subsection{Soil yield displacement}

Fig.7 is variation of maximum lateral displacement with temperature under different soil yield displacement, as figure shows, critical temperature is decreased with soil yield displacement increasing.

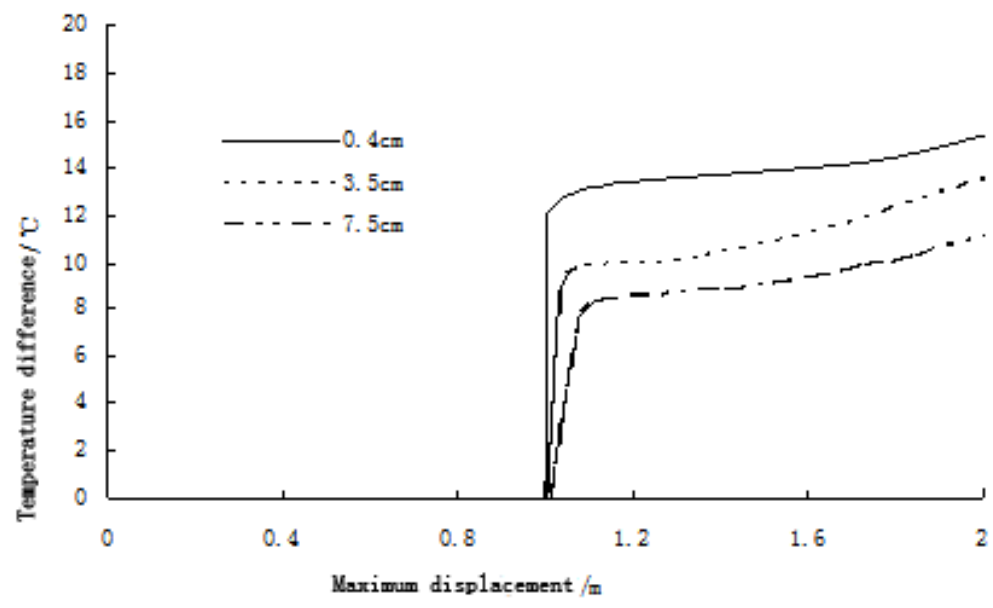

Fig. 7 Temperature vs. maximum lateral displacement for different axial frictional coefficients.

\section{Conclusion}

FEM of pipeline exposed on seabed is built based on ANSYS, interaction between pipeline and seabed is studied, as results show that critical buckling force is increased with lateral frictional coefficient increasing, axial frictional coefficient has little effect on critical buckling force; critical buckling force is reduced with yielding displacement increasing.

\section{Acknowledgements}

The project is supported by project supported by the natural science foundation of Shandong Province (ZR2011DL004), and the Humanity and Social Science Youth foundation of Ministry of Education(13YJCZH114), and National Natural Science Fund Project (41601593). 


\section{References}

[1] Hobbs R E. In-service buckling of heated pipeline [J]. J.Transport Eng, 1984, 110(2):175-189.

[2] Taylor N, Tran V. Experimental and theoretical studies in subsea pipeline buckling[J]. Marine Structures, 1996, 9(2):211-257.

[3] James G A C. A simplified model of upheaval thermal buckling of subsea pipelines[J]. ThinWalled Structures, 1997, 29:59-78.

[4] Ramberg W, Osgood W R. Description of Stress-Strain Curves by Three Parameters[C]. NACA Technical Note, 1943.

[5] Søren H, Bai Y, et al. Use of finite element analysis for local buckling design of pipelines[C]. Proceedings of the 17th International Conference on Offshore Mechanics and Arctic Engineering, Lisbon, Portugal, 1998:1-9.

[6] Lars C. Displacement Control in Lateral Buckling of "Short" Pipelines[C]. Proceedings of the 15th International Offshore and Polar Engineering Conference, Seoul, Korea, 2005: 84-92.

[7] Nader Yoosef-Ghodsi. Analysis of buried pipelines with thermal applications [D]. Canada,University of Alberta,2002.

[8] Enrico T, Luigino V, Erik L. Snaking of submarine pipelines resting on flat sea bottom using finite element method[C]. Proceedings of the 9th International Offshore and Polar Engineering Conference, Brest, France, 1999, 2:34-44.

[9] ANSYS Inc.. ANSYS User' s guide. version10.0. 